\title{
The aetiology of myocardial injury after non-cardiac surgery
}

\author{
R. B. Grobben • W. A. van Klei • D. E. Grobbee • \\ H. M. Nathoe
}

Published online: 20 August 2013

(C) The Author(s) 2013. This article is published with open access at Springerlink.com

\begin{abstract}
Recognition of myocardial injury after non-cardiac surgery is difficult, since strong analgesics (e.g. opioids) can mask anginal symptoms, and ECG abnormalities are subtle or transient. Thorough knowledge of the pathophysiological mechanisms is therefore essential. These mechanisms can be subdivided into four groups: type I myocardial infraction (MI), type II MI, non-ischaemic cardiac pathology, and noncardiac pathology. The incidence of type I MI in patients with a clinical suspicion of perioperative acute coronary syndrome (ACS) is $45-57 \%$. This percentage is higher in patients with a high likelihood of MI such as patients with ST-elevation ACS. Of note, the generalisability of this statement is limited due to significant study limitations. Non-ischaemic cardiac pathology and non-cardiac pathology should not be overlooked as a cause of perioperative myocardial injury (PMI). Especially pulmonary embolism and dysrhythmias are a common phenomenon, and may convey important prognostic value. Implementation of routine postoperative troponin assessment and accessible use of minimally invasive imaging should be considered to provide adequate individualised therapy. Also,
\end{abstract}

\author{
R. B. Grobben $(\bowtie)$ \\ Department of Cardiology and Anaesthesiology, University Medical \\ Center Utrecht, Utrecht, the Netherlands \\ e-mail: r.b.grobben@umcutrecht.nl \\ W. A. van Klei \\ Department of Anaesthesiology, University Medical Center Utrecht, \\ Utrecht, the Netherlands \\ e-mail: w.a.vanklei@umcutrecht.nl \\ D. E. Grobbee \\ Department of Epidemiology, University Medical Center Utrecht, \\ Utrecht, the Netherlands \\ e-mail: d.e.grobbee@umcutrecht.nl \\ H. M. Nathoe \\ Department of Cardiology, University Medical Center Utrecht, \\ Utrecht, the Netherlands \\ e-mail: h.m.nathoe@umcutrecht.nl

addition of preoperative imaging may improve the stratification of high-risk patients who may benefit from preoperative or perioperative interventions.

Keywords Perioperative myocardial injury $\cdot$ Non-cardiac surgery $\cdot$ Perioperative myocardial infarction $\cdot$ Etiology

\section{Introduction}

Annually, over 200 million people undergo major non-cardiac surgery worldwide [1]. Despite technical improvements and increased patient monitoring, these procedures currently remain associated with high mortality and morbidity. Overall 30 -day mortality exceeds $2 \%$, and reaches $6 \%$ in high-risk populations (e.g. certain vascular populations) [2-6]. Over half of these deaths are attributable to adverse cardiovascular events $[3,7]$.

Recognition of an adverse cardiovascular event in the perioperative phase is difficult. Typical anginal symptoms are often masked due of the use of strong analgesics (e.g. opioids), and changes on the electrocardiogram are predominantly subtle or transient $[3,6,8]$. Moreover, the diagnostic value of cardiac biomarkers such as cardiac troponin and creatine kinase muscle and brain isotype (CK-MB) remains controversial in the perioperative period, since factors such as skeletal muscle damage, inflammation and renal insufficiency interfere with serum levels [9]. An adequate and timely diagnosis is therefore frequently delayed, and subsequent therapy may not be initiated at all.

In order to improve early recognition of perioperative myocardial injury (PMI), thorough knowledge of its pathophysiological mechanisms is required. In this article we will elaborate these mechanisms, and review the available evidence concerning perioperative myocardial infarction (MI). 


\section{Aetiology of PMI}

PMI may originate from four distinct pathophysiological mechanisms:

I. MI type $I$ is caused by fissuring or rupturing of an unstable coronary artery atherosclerotic plaque. Consecutive platelet activation and aggregation causes thrombus formation that occludes the vessel, and induces myocardial ischaemia [10]. Surgical patients are especially vulnerable to such adverse events due to a hypercoagulable state in a setting of tachycardia, anaemia, intraoperative hypotension, and postoperative hypertension [8].

II. MI type II is caused by either a haemodynamic equilibrium disturbance combined with the presence of significant coronary artery disease (CAD), a severe coronary vasospasm, or endothelial dysfunction [8]. These mechanisms induce a myocardial oxygen supply-demand mismatch that leads to a more generalised type of myocardial ischaemia than type I [11]. Common perioperative factors that increase myocardial oxygen demand include hypertension, tachycardia and elevated levels of catecholamines [8]. Decreased myocardial oxygen supply is attributable to blood loss, intraoperative hypotension, anaemia, and/or coronary vasospasm [8]. Furthermore, an oxygen supply deficit may develop if the coronary collateral blood supply falls short [12].

III. Non-ischaemic cardiac pathology $[8,10]$. This group can be subdivided into cardiac dysrhythmias, acute nonischaemic cardiomyopathies (e.g. cerebrally or stressinduced), and inflammatory and/or infectious processes. Presence of postoperative electrolyte disturbances, inflammation, hypertension and myocardial ischaemia catalyse the commencement of a dysrhythmia [13, 14]. Non-ischaemic causes other than dysrhythmias are rare, yet can be provoked by certain factors commonly associated with major surgery or trauma (e.g. high stress levels in case of a Takotsubo / stress-induced cardiomyopathy, or a bacteraemia in case of an endocarditis) $[15,16]$.

IV. Non-cardiac causes such as pulmonary embolism (PE). The perioperative phase is associated with prolonged periods of immobilisation in a period of haemodynamic changes. Therefore, ideal circumstances are present for the formation of a venous thromboembolism, and/or a pulmonary embolism $[17,18]$. The incidence of noncardiac causes other than PE is low, and mainly restricted to certain high-risk patients.

A combination of two or more pathophysiological mechanisms is also possible. For instance, a myocardial oxygen supply-demand mismatch (i.e. type II MI) may lead to increased shear stress of an atherosclerotic plaque that consecutively causes the plaque to fissure or rupture (i.e. type I MI induction) $[8,10]$.

\section{Literature}

Angiographic, histological or alternative imaging studies that provide an overview of the prevalence of all four aetiological mechanisms of PMI are currently not available. Therefore, we have to extrapolate data from studies that have focussed on subgroups. Myocardial infarction type I and II will be emphasised, as myocardial ischaemia has been suggested to be the main cause of postoperative morbidity and mortality $[3,8]$.

\section{Type I versus type II myocardial infarction}

The incidence of ST-segment elevation myocardial infarction (STEMI) after non-cardiac surgery ranges from $0.06 \%$ to $1.5 \%$, and is therefore very low $[11,19,20]$. In contrast, transient periods of ST depression are relatively common, as incidences range from $0.6 \%$ to $50 \%$ (i.e. numbers vary based on study population and the use of continuous or interval ECG monitoring) [11, 20, 21]. The ST depressions have historically been associated with type II MI, yet - especially in the perioperative phase - merely provide a suggestion of the underlying pathophysiological mechanism [11]. Routine postoperative measurement of cardiac biomarkers, which are highly sensitive for myocardial damage and provide a broader diagnostic window than a non-continuous ECG, essentially suffers from the same inability to provide evidence concerning the pathophysiological mechanism [19, 22].

Four studies were conducted to assess the incidence of the two types of myocardial infarction after surgery: two necropsy studies and two angiographic studies (Table 1). Appraisal of study relevance and validity is shown in Table 2 , and the results are shown in Table 3.

Dawood et al. [23] performed a histopathological analysis of coronary arteries and myocardium of autopsy heart specimens of patients who suffered either a fatal perioperative MI $(n=42)$, or a non-perioperative MI $(n=25)$. Patients in the former group were eligible for inclusion if they had suffered an intraoperative MI, or a MI $<30$ days of elective or emergency surgery. The latter group was characterised by evidence of MI in a non-surgical phase (i.e. no surgery for at least 3 months prior to their infarction). Diagnostic criteria for MI were based on laboratory biomarkers, clinical parameters (e.g. chest pain and/or pulmonary oedema), and an electrocardiogram (ECG). Histological evidence of plaque rupture and haemorrhage into the plaque cavity or into the lumen was considered direct evidence of disruption of an unstable plaque (type I MI). This was found in $55 \%$ (95\% CI 40-70\%) of patients with a fatal perioperative MI, and in $40 \%$ of patients with a fatal non-perioperative MI. Hence, no significant differences between the two groups were reported regarding type I MI. Intracoronary thrombus was found in $28 \%$. 
Table 1 Study design

\begin{tabular}{llllll}
\hline & Design 1 & Design 2 & Design 3 & Design 4 & $\begin{array}{l}\text { Method } \\
\text { to define } \\
\text { MI }\end{array}$ \\
\hline Dawood & Necropsy $^{\mathrm{a}}$ & Retrospective & Aetiological & Case & $\mathrm{HP}^{\mathrm{e}}$ \\
Cohen & Necropsy $^{\mathrm{b}}$ & Retrospective & Aetiological & Cohort & $\mathrm{HP}^{\mathrm{e}}$ \\
Gualandro & In-vivo $^{\mathrm{c}}$ & Prospective & Aetiological & $\begin{array}{c}\text { Case } \\
\text { control }\end{array}$ & $\mathrm{CAG}^{\mathrm{fgg}}$ \\
Berger & In-vivo $^{\mathrm{d}}$ & Retrospective & Therapeutic & $\begin{array}{c}\text { Cohort } \\
\mathrm{CAG}^{\mathrm{h}}\end{array}$ \\
\hline
\end{tabular}

MI Myocardial infarction

$C A G$ Coronary Angiography

HP Histopathology

${ }^{\text {a }}$ Fatal intraoperative MI, or a MI $<30$ days after non-cardiac surgery versus fatal non-perioperative MI

${ }^{\mathrm{b}}$ Fatal postoperative $\mathrm{MI} \leq 72 \mathrm{~h}$ after non-cardiac surgery

${ }^{\mathrm{c}}$ Suspicion of ACS $<30$ days after non-cardiac surgery versus nonperioperative ACS versus stable CAD

${ }^{\mathrm{d}}$ Referred for CAG because of a suspicion of acute coronary syndrome $<7$ days after non-cardiac surgery

${ }^{\mathrm{e}}$ Histopathological evidence of unstable plaque with disruption

${ }^{\mathrm{f}}$ Complex atherosclerotic plaque morphology based on Goldstein's criteria

${ }^{\mathrm{g}}$ Ambrose type II eccentric lesions

${ }^{\mathrm{h}}$ Intracoronary evidence of thrombus

Cohen et al. [24] performed a necropsy study that assessed the underlying pathophysiological mechanism of a fatal postoperative MI $<72 \mathrm{~h}$ of elective surgery $(n=26)$. The presence of plaque cap fissuring (i.e., disruption of a fibrous cap), plaque haemorrhage, or luminal thrombus containing plaque elements (e.g., cholesterol crystals and lipid-laden macrophages) was considered evidence of plaque rupture (i.e. type I MI). This was present in $46 \%$ (95 \% CI $27-$ $67 \%$ ) of patients. Thrombus was found in $35 \%$.

Gualandro et al. [25] performed a prospective angiographic study to assess the cardiac pathophysiological mechanism in 120 patients with a clinical suspicion of acute coronary syndrome (ACS)) within 30 days after noncardiac surgery. Results were compared with angiographic data from 120 non-surgical patients with spontaneous ACS, and 240 patients with stable CAD. Ambrose type II eccentric lesions (Table 4) and complex morphological features (Table 5) were considered evidence of type I MI $[26,27]$. In the postoperative group, these incidences were $45 \%(95 \%$ CI $36-54 \%)$ and $57 \%$ (95\% CI 48 $66 \%$ ), respectively. These numbers were significantly higher in the non-perioperative ACS group (57 \% and $79 \%$, respectively), and significantly lower in the stable CAD group ( $16 \%$ and $32 \%$, respectively). Thrombus was reported in $7.5 \%$, $32.5 \%$, and $8.8 \%$ for perioperative ACS, spontaneous ACS, and stable $\mathrm{CAD}$, respectively.

Berger et al. [28] performed a retrospective therapeutic study to assess the best immediate intervention in 48 patients referred for coronary angiography because of a suspicion of acute coronary syndrome within 7 days after non-cardiac surgery. All patients had ischaemic chest pain $\geq 30 \mathrm{~min}$ or haemodynamic instability combined with typical ECG changes. Evidence of type I MI was present in $90 \%(95 \%$ CI $81 \%-98 \%$ ) of patients, and intracoronary thrombus was found in $63 \%$.

Table 2 Appraisal

\begin{tabular}{|c|c|c|c|c|c|c|c|c|c|}
\hline & \multicolumn{4}{|c|}{ Generalisability } & \multicolumn{5}{|l|}{ Validity } \\
\hline & Study design & Domain & Determinant & Outcome & Blinding & Baseline & Standard & Routine screening & Missing data \\
\hline Dawood & $+/-$ & + & + & + & - & - & + & - & $?$ \\
\hline Cohen & $+/-$ & + & + & + & + & - & + & - & $?$ \\
\hline Gualandro & + & - & + & + & + & + & + & - & + \\
\hline Berger & - & + & - & - & - & $+/-$ & $?$ & - & $?$ \\
\hline
\end{tabular}

Study design Prospective aetiological study $(+)$; retrospective aetiological study $(+/-)$; other $(-)$

Domain Patients with a perioperative ACS: $<7$ days $(+) ;>7$ days $(-)$

Determinant Methods to define type I MI well described: yes (+); no (-)

Outcome Angiographic or histopathological data well described: yes (+); no (-)

Blinding Blinding of assessor for clinical characteristics: present (+); absent (-); incomplete documentation (?)

Baseline Adequate description of angina, relevant comorbidities and prior cardiac adverse events, ECG characteristics, relevant drug use: $4 / 4$ determinants $(+) ; 3$ determinants $(+/-),<3$ determinants $(-)$

Standard Use of angiographic or histopathological data in accordance to objectified principals: yes (+); no (-); insufficient description (?)

Routine screening Use of routine diagnostic screening (routine ECG assessment, continuous ECG monitoring or routine biomarker assessment) : yes (+); no $(-)$

Missing data $<10 \%(+) ;>10 \%(-)$; not mentioned (?) 
Table 3 Results

\begin{tabular}{|c|c|c|c|c|c|c|c|c|c|c|}
\hline & \multicolumn{5}{|c|}{ Clinical signs } & \multicolumn{5}{|c|}{ Angiographic / histopathological data } \\
\hline & $\begin{array}{l}\text { Sample } \\
\text { size (n) }\end{array}$ & $\begin{array}{l}\text { Clinical signs } \\
\text { of MI }\end{array}$ & Angina & STE-ACS & NSTE-ACS & $\begin{array}{l}\text { Average time } \\
\text { to } \mathrm{CAG}\end{array}$ & $\begin{array}{l}\text { Multivessel } \\
\text { disease }\end{array}$ & No CAD & MI type I & $\begin{array}{l}\text { Presence of } \\
\text { thrombus }\end{array}$ \\
\hline Dawood & 42 & $100 \%$ & - & - & - & - & $95.3 \%$ & $4.7 \%$ & $55 \%{ }^{\mathrm{a}}$ & $28 \%$ \\
\hline Cohen & 26 & $42.3 \%$ & - & - & - & - & $88 \%$ & $11.5 \%$ & $46 \%^{\mathrm{a}}$ & $35 \%$ \\
\hline Gualandro & 120 & $100 \%$ & $40.7 \%$ & $5.8 \%$ & $65.1 \%$ & 5.5 days & - & $5.8 \%$ & $\begin{array}{l}45 \%{ }^{\mathrm{b}} \\
57 \%^{\mathrm{c}}\end{array}$ & $7 \%$ \\
\hline Berger & 48 & $100 \%$ & $100 \%$ & $68.8 \%$ & $31.3 \%$ & 0.5 days & $87.5 \%$ & $0 \%$ & $90 \%{ }^{\mathrm{d}}$ & $63 \%$ \\
\hline
\end{tabular}

$n$ Number of included patients with perioperative MI

$M I$ Myocardial infarction

STE-ACS ST-segment-elevation acute coronary syndrome

NSTE-ACS Non ST-segment-elevation acute coronary syndrome

$C A G$ Coronary angiography

$C A D$ Coronary artery disease

- Data not available

${ }^{a}$ Histopathological evidence of unstable plaque with disruption

${ }^{\mathrm{b}}$ Ambrose type II eccentric lesions

${ }^{\mathrm{c}}$ Complex atherosclerotic plaque morphology based on Goldstein's criteria

${ }^{\mathrm{d}}$ Unknown

\section{Non-ischaemic cardiac and extra-cardiac pathophysiological mechanisms of PMI}

PMI is not exclusively caused by myocardial infarction. It can also be a result of non-ischaemic cardiac pathology (e.g. dysrhythmias, acute non-ischaemic cardiomyopathies, and inflammatory and/or infectious processes) and/or extracardiac pathology (i.e. predominantly pulmonary embolisms).

Non-ischaemic cardiac pathology

The pathophysiology of the non-ischaemic causes of PMI can be subdivided into cardiac dysrhythmias, acute non-ischaemic cardiomyopathies (e.g. cerebrally or stressinduced), and inflammatory and/or infectious processes.

Table 4 Ambrose criteria [26]

\begin{tabular}{ll}
\hline Type & Characteristics \\
\hline $\begin{array}{l}\text { Concentric } \\
\text { Type I eccentric }\end{array}$ & $\begin{array}{c}\text { Symmetric and smooth narrowing } \\
\text { Asymmetric stenosis with smooth borders and } \\
\text { a broad neck } \\
\text { Aypymmetric stenosis in the form of a convex } \\
\text { intraluminal obstruction with a narrow neck due } \\
\text { to one or more overhanging edges or irregular } \\
\text { or scalloped borders, or both }\end{array}$ \\
$\begin{array}{c}\text { Multiple } \\
\text { irregularities or more serial, closely spaced narrowing or } \\
\text { severe diffuse irregularities within a vessel }\end{array}$ \\
\hline
\end{tabular}

A cardiac dysrhythmia after non-cardiac surgery is a common phenomenon [14, 29]. Yet, available evidence is predominantly based on retrospective studies using noncontinuous ECG monitoring. These studies have designated atrial fibrillation (AF) as the most frequent arrhythmia, affecting an average of 3-4\% of patients undergoing noncardiac surgery $[14,29]$. An additional 3-4\% develop a supraventricular tachycardia (SVT) other than AF. All dysrhythmias are associated with a worse outcome; a large multicentre trial reported an increased risk of mortality (adjusted odds ratio 1.72; $95 \%$ CI, 1.59-1.86), longer hospital stays (adjusted relative difference, $+24.0 \%$; $95 \%$ CI 21.5-26.5\%), and increased hospitalisation costs (adjusted difference, $+\$ 4177 ; 95 \%$ CI $\$ 3764-4590)$. Perioperative mortality directly resulting from a dysrhythmia is seldom reported after non-cardiac surgery, and its incidence is

Table 5 Criteria for complex lesions - Goldstein et al. [27]

\footnotetext{
- An intraluminal filling defect consistent with thrombus, defined as abrupt vessel cutoff with persistence of contrast, or an intraluminal filling defect in a vessel within or adjacent to a stenotic region with surrounding homogeneous contrast opacification

- Plaque ulceration, defined by the presence of contrast and hazy contour beyond the vessel lumen;

- Plaque irregularity (haziness), defined by irregular margins or overhanging edges

- Impaired flow (TIMI flow $<3$, except lesions characteristic of chronic total occlusion, identified as tapering lesions with multiple fine collaterals)
} 
pragmatically believed to be lower than $0.6 \%$ [30]. Factors associated with an increased risk of developing a perioperative dysrhythmia include age, significant valvular disease, congestive heart failure, prior dysrhythmias, myocardial ischaemia, and electrolyte disturbances [14]. Also, a (pro-dysrhythmogenic) myocardial contusion should be considered in patients who have experienced blunt thoracic trauma prior to hospitalisation [31].

A perioperative cerebrally or stress-induced cardiomyopathy can be provoked by an acute cerebrovascular event (predominantly subarachnoid haemorrhage) and/or high preoperative stress levels [15, 32]. Especially stress-induced cardiomyopathies may clinically present as an acute coronary syndrome, as characteristic ECG signs of a myocardial infarction may be present, and cardiac biomarkers may be elevated [33]. The incidence of such cardiomyopathies is low, and current evidence mainly consists of case reports [15, 32, 34].

Postoperative infections and systemic inflammatory response syndromes (SIRS) are a frequent complication of surgery in general [16]. Yet, inflammation or infection of cardiac structures (i.e. myocarditis, pericarditis or endocarditis) after non-cardiac procedures is rare, and chiefly restricted to patients with prosthetic heart valves [35]. In those patients, pathogens such as Staphylococcus aureus are capable of causing acute valvular failure, which is associated with a very poor prognosis [36].

\section{Extracardiac pathology}

The perioperative phase is associated with prolonged periods of immobilisation and a hypercoagulable state. Therefore, ideal conditions are present for the formation of a venous thromboembolism and a pulmonary embolism (PE). Studies regarding postoperative PE have mainly focussed on orthopaedic and oncological populations, in which the incidence of symptomatic and fatal PE is approximately $1 \%$ and $0.1 \%$, respectively $[17,18,37]$. Asymptomatic $\mathrm{PE}$ is observed in $2-$ $4 \%$, and is therefore at least twice as common as its symptomatic counterpart $[38,39]$. Of note, these numbers are based on studies using a ventilation/perfusion (V/Q) scan. Using computed tomography pulmonary angiography (CTPA), which has better spatial resolution and diagnostic accuracy than a ventilation-perfusion (V/Q) scan, small (asymptomatic) perfusion defects can be detected more accurately [40, 41]. If these perfusion defects are taken into account, the incidence of asymptomatic PE after non-cardiac surgery is expected to exceed $4 \%[37,42,43]$. However, the clinical relevance of small - mostly asymptomatic - perfusion defects has not yet been fully determined [44].

Other acute extracardiac causes such as a postoperative aortic dissection are very rare after non-cardiac surgery, and should primarily be suspected in patients with relevant extracardiac morbidities, or a high cardiovascular risk profile (e.g. those with a (familial) history of aortic aneurysms/ dissections and/or connective tissue disorders such as Marfan syndrome or Ehlers-Danlos syndrome) [45]. Postoperative hypertension may - in these patients - lead to an increased risk of an aortic dissection [45].

\section{Discussion}

The incidence of type I MI in patients who suffer a fatal perioperative MI ranges from 45 to $55 \%$ (Table 3). This is not significantly different from the non-surgical patients with fatal MI or sudden cardiac death, in which type I MI represents $50-80 \%[23,46,47]$. In patients with a clinical suspicion of acute coronary syndrome after non-cardiac surgery, type I MI is observed in $45-57 \%$ (Table 3 ). This percentage is much higher in patients with a high likelihood of MI, such as patients presenting with ST-elevation ACS [28]. Of note, the validity and generalisability of these results are retained due a number of limitations (Table 2).

First, the study populations and designs of the aforementioned studies are not interchangeable (Table 1). Dawood studied 42 patients with $\mathrm{MI}<30$ days of elective or emergency surgery using a case-control design, whilst Cohen studied 26 patients with $\mathrm{MI}<72 \mathrm{~h}$ after elective surgery using a cohort design. Of note, the study populations and designs of the angiographic studies were even less interchangeable: Gualandro performed a prospective aetiological study that assessed the incidence of type I MI in patients suspected of ACS, whilst Berger performed a retrospective therapeutic study to assess the best immediate invasive strategy in patients with an (almost certain) perioperative MI (Table 1).

Second, the methods to define MI (Table 1) are heterogenic; Dawood and Cohen used a histopathological analysis (i.e. the gold standard), whilst the in-vivo studies used conventional coronary angiography (CAG). A CAG has high diagnostic accuracy for MI, yet a number of limitations have to be recognised [48, 49]. For instance, its interpretation is primarily based on the assessment of significant coronary lesions. Yet, a significant stenosis is not a necessity for a type I MI, since normal angiographic findings have been reported in 4-31\% of non-surgical patients with acute MI [48, 49]. In those cases, outward remodelling of an unstable atherosclerotic plaque may have only caused a slight, or even a non-existent coronary lumen reduction [50]. The share of type I MI may thus be underestimated.

Third, the incidence of clinical signs and symptoms suggestive of MI varies between studies (Table 3); Cohen and Gualandro reported incidences of $42.5 \%$ and $41 \%$, respectively. Berger reported anginal symptoms in $100 \%$, and Dawood did not provide any numbers. The high incidence reported by Berger reflects its methodological shortcomings to 
Table 6 Useful diagnostic modalities to determine the pathophysiology of PMI

\begin{tabular}{|c|c|c|}
\hline Type & & Characteristics \\
\hline \multirow[t]{2}{*}{ CAG } & Pro & $\begin{array}{l}\text { Best diagnostic accuracy for in-vivo } \\
\text { assessment of myocardial infarction. Gold } \\
\text { standard. Option of immediate assessment } \\
\text { of clinical significance of coronary stenoses } \\
\text { with FFR. Also, immediate PCI } \\
\text { is possible }\end{array}$ \\
\hline & Con & $\begin{array}{l}\text { Invasive, risk of iatrogenic coronary } \\
\text { dissection. Not attractive in the early } \\
\text { postoperative phase. Use of contrast fluid. } \\
\text { Radiation exposure. Expensive }\end{array}$ \\
\hline IVUS & Pro & $\begin{array}{l}\text { Assessment of both coronary lumen, and } \\
\text { plaque morphology (e.g. atheroma). } \\
\text { Assessment of unstable/vulnerable } \\
\text { plaques; plaque rupture, erosion, and } \\
\text { intracoronary thrombus. Especially useful } \\
\text { in situations in which angiographic imaging } \\
\text { is considered unreliable, such overlapping } \\
\text { vessels that cannot be adequately assessed } \\
\text { with CAG, and in case of outward plaque } \\
\text { remodelling }\end{array}$ \\
\hline
\end{tabular}

Con Expensive, invasive, time-consuming. Only performed by specialised angiographers. Use of contrast fluid. Radiation exposure. Measurement difficulties in case of bifurcations

OCT

Echocardiogram Pro Assessment of myocardial segmental wall kinetics, right ventricular kinetics, and valvular apparatus. No radiation exposure

Con Coronary and pulmonary arteries no visualised, variable image quality

CCTA Pro Number and degree of coronary artery stenoses, coronary calcifications, aspect of coronary plaque. Possibility of obtaining coronary artery calcium score

Con Artefacts due to calcifications. Pulmonary arteries not or only partially visualised. Overestimation of stenosis degree in calcified plaques (blooming). Use of contrast fluid. Radiation exposure

CTPA

Pro Assessment of pulmonary arteries. Highly sensitive for PE

Con Diagnosis of small perfusion defects with unknown prognostic value. Coronaries and heart are not accurately visualised. Use of contrast fluid. Radiation exposure
Table 6 (continued)

\begin{tabular}{|c|c|c|}
\hline Type & & Characteristics \\
\hline \multirow[t]{2}{*}{ Cardiac MRI } & Pro & $\begin{array}{l}\text { Highly sensitive for MI. Assessment of } \\
\text { myocardial contraction, and segmental wall } \\
\text { kinetics. No radiation exposure }\end{array}$ \\
\hline & Con & $\begin{array}{l}\text { Coronaries and pulmonary arteries not or only } \\
\text { partially visualised. Expensive, time- } \\
\text { consuming. Use of gadolinium }\end{array}$ \\
\hline \multirow[t]{2}{*}{$\begin{array}{l}\text { Cardiac adenosine } \\
\text { stress MRI }\end{array}$} & Pro & $\begin{array}{l}\text { Highly sensitive for MI. Assessment of } \\
\text { clinical significance of coronary stenoses } \\
\text { by generating chemically-induced } \\
\text { myocardial ischaemia. }\end{array}$ \\
\hline & Con & $\begin{array}{l}\text { Coronaries and pulmonary arteries not or only } \\
\text { partially visualised. Expensive, time- } \\
\text { consuming. Use of gadolinium. Use of } \\
\text { adenosine. Limited availability }\end{array}$ \\
\hline
\end{tabular}

$P M I$ Perioperative Myocardial Injury

$C A G$ Coronary Angiography

$P C I$ Percutaneous Coronary Intervention

FFR Fractional Flow Reserve

IVUS Intravascular ultrasound

OCT Optical Coherence Tomography

CCTA Coronary Computed Tomography Angiography

$P E$ Pulmonary Embolism

CTPA Computed Tomography Pulmonary Angiography

MRI Magnetic Resonance Imaging

answer our clinical question. Moreover, it underlines the fact that its population does not fully correspond to the average population with a perioperative MI. The incidences reported by Gualandro appear to be consistent with a large prospective study that reported an incidence of ischaemic symptoms in patients with perioperative non-fatal MI of $38.1 \%$ [4]. However, Gualandro did not perform routine assessments of cardiac biomarkers and/or continuous ECG monitoring. PMI could therefore have been missed in patients using strong analgesics. As a result, a clinically moderate group was studied, and the incidence of type I may have been overestimated. This suggestion is supported by high numbers of multi- or three-vessel disease (Table 3). Of note, the overestimation may have been somewhat tempered by missing data from critically ill patients who died before the angiography was performed [25].

Fourth, the incidence of coronary thrombus differs between the angiographic studies; Gualandro reported $7 \%$ compared to $63 \%$ reported by Berger (Table 3). The low incidence found by Gualandro may have been related to the long interval between the MI and the angiography (average delay of 5.5 days versus 0.5 days in Gualandro versus Berger, respectively), the use of statins, antiplatelet and anticoagulant agents, and a lower likelihood of a type I MI than Berger. All of these factors may have contributed to (spontaneous) thrombolysis. The diagnostic delay can be 
explained by the undesirability of coronary angiography in the (very) early postoperative period, which is a result of its invasive nature, and the necessity of administration of antiplatelet agents and heparin.

Furthermore, the share of non-ischaemic and extracardiac pathology may not be overlooked as a cause of PMI. Especially cardiac dysrhythmias and pulmonary embolisms are common after noncardiac surgery, yet-as with perioperative MI-frequently remain asymptomatic. Current studies tend to be biased toward symptomatic cases that represent a population with a worse prognosis. In case of pulmonary embolisms, this is underlined by a high incidence of impaired right ventricular function, which correlates with a higher risk of death within 3 months (hazard ratio 2.2 (95\% CI 1.4-3.4)) [17]. Increased accessibility of computed tomography angiography (CTA) will lead to an increased recognition of (asymptomatic) PE [51]. Yet, the clinical relevance of small asymptomatic perfusion defects detected by CTA is currently unknown and should be evaluated in the near future [44].

Clinical signs and ECGs cannot guarantee early recognition of PMI, and the implementation of standard continuous ECG monitoring is difficult. Additional screening therefore appears to be indispensable. This creates a window of opportunity for routine postoperative assessment of biomarkers such as cardiac troponin (cTn), which has recently shown to be a strong and independent predictor of short- and intermediate-term mortality [3, 19, 22]. Its diagnostic interpretation, however, can be troublesome due to the interference of renal dysfunction, cerebral pathology and inflammation [9]. cTn can therefore not be used as hard evidence of myocardial injury. Yet, it can be used to stratify patients who could benefit from additional anticoagulant therapy or a percutaneous coronary intervention (PE/MI or MI, respectively). Non and minimally invasive modalities (i.e. CTA, MRA and echocardiography) should be accessibly used in the early postoperative phase to avoid the use of superfluous anticoagulant and antiplatelet agents that are a necessity during CAG. The most valuable diagnostic modality should be individually determined based on the patient's type of surgery, medical history, clinical presentation and comorbidities (Table 6).

Furthermore, the additive value of preoperative cardiac imaging needs to be evaluated, since current clinical prediction models convey prognostic value, yet frequently fail to adequately predict PMI $[52,53]$. Addition of modalities such as a (coronary) CTA may improve prognostic values, and could lead to a better stratification of patients who benefit from additional preoperative or perioperative interventions [54]. The relevance of such a selection is underlined by the fact that a reduction in cardiac events can be counterbalanced by an increased risk of cerebrovascular pathology or bleeding (e.g. in case of antiplatelet agents) $[4,55]$.

\section{Conclusion}

Recognition of perioperative myocardial injury after noncardiac surgery is difficult due to the masking of angina, and the subtlety or transiency of ECG abnormalities. Thorough knowledge of the pathophysiologic mechanisms is therefore essential. These mechanisms can be subdivided into four groups: type I MI, type II MI, non-ischaemic cardiac pathology, and non-cardiac pathology.

In patients suspected of a perioperative acute coronary syndrome. The incidence of type I MI is $45-57 \%$; this percentage is higher in those with a high likelihood of MI such as patients with ST-elevation ACS. Of note, the generalisability of this statement is limited due to significant study limitations.

Non-ischaemic cardiac pathology and non-cardiac pathology should not be overlooked as a cause of PMI. Especially pulmonary embolisms and dysrhythmias are a common phenomenon, and may convey important prognostic value. Implementation of routine postoperative troponin assessment and accessible use of minimally invasive imaging should be considered to provide adequate individualised therapy. Also, the addition of preoperative imaging may also improve stratification of high-risk patients that will benefit from preor perioperative interventions.

\section{Funding None.}

Conflict of interests None declared.

Open Access This article is distributed under the terms of the Creative Commons Attribution License which permits any use, distribution, and reproduction in any medium, provided the original author(s) and the source are credited.

\section{References}

1. Weiser TG, Regenbogen SE, Thompson KD, et al. An estimation of the global volume of surgery: a modelling strategy based on available data. Lancet. 2008;372(9633):139-44.

2. Pearse RM, Moreno RP, Bauer P, et al. Mortality after surgery in Europe: a 7 day cohort study. Lancet. 2012;380(9847):1059-65.

3. Vascular Events In Noncardiac Surgery Patients Cohort Evaluation (VISION) Study Investigators, Devereaux PJ, Chan MT, et al. Association between postoperative troponin levels and 30-day mortality among patients undergoing noncardiac surgery. JAMA. 2012; 307(21):2295-304.

4. POISE Study Group, Devereaux PJ, Yang H, et al. Effects of extended-release metoprolol succinate in patients undergoing noncardiac surgery (POISE trial): a randomised controlled trial. Lancet. 2008;371(9627):1839-47.

5. Fleisher LA, Eagle KA, Shaffer T, et al. Perioperative- and long-term mortality rates after major vascular surgery: the relationship to preoperative testing in the medicare population. Anesth Analg. 1999;89(4):849-55. 
6. Mangano DT, Goldman L. Preoperative assessment of patients with known or suspected coronary disease. N Engl J Med. 1995;333(26): 1750-6.

7. Noordzij PG, Poldermans D, Schouten O, et al. Postoperative mortality in the Netherlands: a population-based analysis of surgery-specific risk in adults. Anesthesiology. 2010;112(5):1105-15.

8. Landesberg G, Beattie WS, Mosseri M, et al. Perioperative myocardial infarction. Circulation. 2009;119(22):2936-44.

9. Khan IA, Tun A, Wattanasauwan N, et al. Elevation of serum cardiac troponin I in noncardiac and cardiac diseases other than acute coronary syndromes. Am J Emerg Med. 1999;17(3):225-9.

10. Thygesen K, Alpert JS, Jaffe AS, et al. Third universal definition of myocardial infarction. J Am Coll Cardiol. 2012;60(16):1581-98.

11. Landesberg G, Mosseri M, Zahger D, et al. Myocardial infarction after vascular surgery: the role of prolonged stress-induced, ST depression-type ischemia. J Am Coll Cardiol. 2001;37(7):1839-45.

12. Ellis SG, Hertzer NR, Young JR, et al. Angiographic correlates of cardiac death and myocardial infarction complicating major nonthoracic vascular surgery. Am J Cardiol. 1996;77(12):1126-8.

13. Creswell LL. Postoperative atrial arrhythmias: risk factors and associated adverse outcomes. Semin Thorac Cardiovasc Surg. 1999;11(4):303-7.

14. Bhave PD, Goldman LE, Vittinghoff E, et al. Incidence, predictors, and outcomes associated with postoperative atrial fibrillation after major noncardiac surgery. Am Heart J. 2012;164(6):918-24.

15. Hakeem A, Marks AD, Bhatti S, et al. When the worst headache becomes the worst heartache! Stroke. 2007;38(12):3292-5.

16. Rangel-Frausto MS, Pittet D, Costigan M, et al. The natural history of the systemic inflammatory response syndrome (SIRS). A prospective study. JAMA. 1995;273(2):117-23.

17. Goldhaber SZ, Visani L, De Rosa M. Acute pulmonary embolism: clinical outcomes in the International Cooperative Pulmonary Embolism Registry (ICOPER). Lancet. 1999;353(9162):1386-9.

18. Martino MA, Borges E, Williamson E, et al. Pulmonary embolism after major abdominal surgery in gynecologic oncology. Obstet Gynecol. 2006;107(3):666-71.

19. Van Waes JA, Nathoe HM, de Graaff JC, et al. Myocardial injury after noncardiac surgery and its association with short-term mortality. Circulation. 2013;127(23):2264-71.

20. Mangano DT, Browner WS, Hollenberg M, et al. Association of perioperative myocardial ischemia with cardiac morbidity and mortality in men undergoing noncardiac surgery. The Study of Perioperative Ischemia Research Group. New Engl J Med. 1990; 323:1781-8.

21. Böttiger BW, Motsch J, Teschendorf P, et al. Postoperative 12-lead ECG predicts peri-operative myocardial ischaemia associated with myocardial cell damage. Anaesthesia. 2004;59(11):1083-90.

22. Levy M, Heels-Ansdell D, Hiralal R, et al. Prognostic value of troponin and creatine kinase muscle and brain isoenzyme measurement after noncardiac surgery: a systematic review and metaanalysis. Anesthesiology. 2011;114(4):796-806.

23. Dawood MM, Gutpa DK, Southern J, et al. Pathology of fatal perioperative myocardial infarction: implications regarding pathophysiology and prevention. Int J Cardiol. 1996;57(1):37-44.

24. Cohen MC, Aretz TH. Histological analysis of coronary artery lesions in fatal postoperative myocardial infarction. Cardiovasc Pathol. 1999;8(3):133-9.

25. Gualandro DM, Campos CA, Calderaro D, et al. Coronary plaque rupture in patients with myocardial infarction after noncardiac surgery: frequent and dangerous. Atherosclerosis. 2012;222(1):191-5.

26. Ambrose JA, Winters SL, Stern A, et al. Angiographic morphology and the pathogenesis of unstable angina pectoris. J Am Coll Cardiol. 1985;5(3):609-16.

27. Goldstein JA, Demetriou D, Grines CL, et al. Multiple complex coronary plaques in patients with acute myocardial infarction. $\mathrm{N}$ Engl J Med. 2000;343(13):915-22.
28. Berger PB, Bellot V, Bell MR, et al. An immediate invasive strategy for the treatment of acute myocardial infarction early after noncardiac surgery. Am J Cardiol. 2001;87(9):1100-2. A6, A9.

29. Polanczyk CA, Goldman L, Marcantonio ER, et al. Supraventricular arrhythmia in patients having noncardiac surgery: clinical correlates and effect on length of stay. Ann Intern Med. 1998;129(4):27985.

30. Wiesbauer F, Schlager O, Domanovits H, et al. Perioperative beta-blockers for preventing surgery-related mortality and morbidity: a systematic review and meta-analysis. Anesth Analg. 2007; 104(1):27-41.

31. Fabian TC, Mangiante EC, Patterson CR, et al. Myocardial contusion in blunt trauma: clinical characteristics, means of diagnosis, and implications for patient management. J Trauma. 1988;28(1):50-7.

32. Das M, Gonsalves S, Saha A, et al. Acute subarachnoid haemorrhage as a precipitant for takotsubo cardiomyopathy: a case report and discussion. Int J Cardiol. 2009;132(2):283-5.

33. Abe Y, Kondo M, Matsuoka R, et al. Assessment of clinical features in transient left ventricular apical ballooning. J Am Coll Cardiol. 2003;41(5):737-42.

34. Konrad FM, Unertl KE, Schroeder TH. Takotsubo cardiomyopathy after cerebral aneurysm rupture. J Neurosurg Anesthesiol. 2010; 22(2):181-2.

35. Biteker M, Tekkeșin Aİ, Can MM, et al. Outcome of noncardiac and nonvascular surgery in patients with mechanical heart valves. Am J Cardiol. 2012;110(4):562-7.

36. Gottlieb GS, Fowler Jr VG, Kong LK, et al. Staphylococcus aureus bacteremia in the surgical patient: a prospective analysis of 73 postoperative patients who developed Staphylococcus aureus bacteremia at a tertiary care facility. J Am Coll Surg. 2000;190(1):50-7.

37. Gandhi R, Salonen D, Geerts WH, et al. A pilot study of computed tomography-detected asymptomatic pulmonary filling defects after hip and knee arthroplasties. J Arthroplasty. 2012;27(5):730-5.

38. Balderston RA, Graham TS, Booth Jr RE, et al. The prevention of pulmonary embolism in total hip arthroplasty. Evaluation of low-dose warfarin therapy. J Arthroplasty. 1989;4(3):217-21.

39. Wolf LR, Hozack WJ, Balderston RA, et al. Pulmonary embolism. Incidence in primary cemented and uncemented total hip arthroplasty using low-dose sodium warfarin prophylaxis. J Arthroplasty. 1992; 7(4):465-70.

40. The PIOPED Investigators. Value of the ventilation/perfusion scan in acute pulmonary embolism. Results of the prospective investigation of pulmonary embolism diagnosis (PIOPED). JAMA. 1990; 263(20):2753-9.

41. Stein PD, Fowler SE, Goodman LR, et al. Multidetector computed tomography for acute pulmonary embolism. $\mathrm{N}$ Engl $\mathrm{J}$ Med. 2006;354(22):2317-27.

42. Okadome M, Saito T, Miyahara D, et al. Postoperative pulmonary embolism including asymptomatic cases in gynecologic oncology. Int J Gynecol Cancer. 2011;20(4):655-63.

43. Watanabe H, Sekiya H, Kariya Y, et al. The incidence of venous thromboembolism before and after total knee arthroplasty using 16row multidetector computed tomography. J Arthroplasty. 2011; 26(8):1488-93.

44. Anderson DR, Kahn SR, Rodger MA, et al. Computed tomographic pulmonary angiography vs ventilation-perfusion lung scanning in patients with suspected pulmonary embolism: a randomized controlled trial. JAMA. 2007;298(23):2743-53.

45. Hiratzka LF, Bakris GL, Beckman JA, et al. ACCF/AHA/AATS/ ACR/ASA/SCA/SCAI/SIR/STS/SVM Guidelines for the diagnosis and management of patients with thoracic aortic disease. A Report of the American College of Cardiology Foundation/American Heart Association Task Force on Practice Guidelines, American Association for Thoracic Surgery, American College of Radiology,American Stroke Association, Society of Cardiovascular Anesthesiologists, Society for Cardiovascular Angiography and Interventions, Society of 
Interventional Radiology, Society of Thoracic Surgeons,and Society for Vascular Medicine. J Am Coll Cardiol. 2010;55(14):e27-e129.

46. Davies MJ, Thomas A. Thrombosis and acute coronary-artery lesions in sudden cardiac ischemic death. N Engl J Med. 1984;310(18): $1137-40$.

47. Qiao JH, Fishbein MC. The severity of coronary atherosclerosis at sites of plaque rupture with occlusive thrombosis. J Am Coll Cardiol. 1991;17(5):1138-42.

48. Bugiardini R, Bairey Merz CN. Angina with "normal" coronary arteries: a changing philosophy. JAMA. 2005;293(4):477-84.

49. Hochman JS, Tamis JE, Thompson TD, et al. Sex, clinical presentation, and outcome in patients with acute coronary syndromes. Global Use of Strategies to Open Occluded Coronary Arteries in Acute Coronary Syndromes IIb Investigators. N Engl J Med. 1999;341(4):226-32.

50. Mann JM, Davies MJ. Vulnerable plaque. Relation of characteristics to degree of stenosis in human coronary arteries. Circulation. 1996;94(5):928-31.
51. Parvizi J, Smith EB, Pulido L, et al. The rise in the incidence of pulmonary embolus after joint arthroplasty: is modern imaging to blame? Clin Orthop Relat Res. 2007;463:107-13.

52. Ford MK, Beattie WS, Wijeysundera DN. Systematic review: prediction of perioperative cardiac complications and mortality by the revised cardiac risk index. Ann Intern Med. 2010;152(1):26-35.

53. Lee TH, Marcantonio ER, Mangione CM, et al. Derivation and prospective validation of a simple index for prediction of cardiac risk of major noncardiac surgery. Circulation. 1999;100(10):10439.

54. Ahn JH, Park JR, Min JH, et al. Risk stratification using computed tomography coronary angiography in patients undergoing intermediate-risk noncardiac surgery. J Am Coll Cardiol. 2013;61(6): 661-8.

55. Marcos EG, Da Fonseca AC, Hofma SH. Bridging therapy for early surgery in patients on dual antiplatelet therapy after drug-eluting stent implantation. Neth Heart J. 2011;19(10):412-7. 